Donor-specific HLA antibodies in predicting crossmatch outcome : Comparison of three different laboratory techniques

Peräsaari, J. P.

2018-02

Peräsaari , J P , Jaatinen , T \& Merenmies , J 2018 , ' Donor-specific HLA antibodies in predicting crossmatch outcome : Comparison of three different laboratory techniques ' , Transplant Immunology, vol. 46 , pp. 23-28 . https://doi.org/10.1016/j.trim.2017.11.002

http://hdl.handle.net/10138/300963

https://doi.org/10.1016/j.trim.2017.11.002

publishedVersion

Downloaded from Helda, University of Helsinki institutional repository.

This is an electronic reprint of the original article.

This reprint may differ from the original in pagination and typographic detail.

Please cite the original version. 


\title{
Donor-specific HLA antibodies in predicting crossmatch outcome: Comparison of three different laboratory techniques
}

\author{
J.P. Peräsaari ${ }^{\mathrm{a}, *}$, T. Jaatinen ${ }^{\mathrm{a}}$, J. Merenmies $^{\mathrm{b}}$ \\ ${ }^{a}$ Histocompatibility Testing Laboratory, Finnish Red Cross Blood Service, Helsinki, Finland \\ ${ }^{\mathrm{b}}$ Children's Hospital, University of Helsinki and Helsinki University Hospital, Helsinki, Finland
}

\section{A R T I C L E I N F O}

\section{Keywords:}

Crossmatch

Donor-specific antibodies

HLA antibodies

Kidney transplantation

Solid-phase assay

\begin{abstract}
A B S T R A C T
The virtual crossmatch, which is based on single antigen bead technology, is used in the prediction of crossmatch results. However, this assay differs in sensitivity and specificity from crossmatch methods. In our study, the results of physical crossmatches, performed with three different methods, were assessed against virtual crossmatch results. The aim was to determine the potential cut-off values for donor specific antibodies (DSA) that would predict the crossmatch results obtained by different methods. The results of different crossmatch techniques were correlated with the virtual crossmatch. The receiver operating characteristic (ROC) analysis revealed the Flow cytometric crossmatch (FCXM) and Luminex crossmatch (LXM) to be the most accurate, with area under curve (AUC) values of 0.861 and 0.805 , respectively. While we found that the virtual crossmatch correlated well with all the crossmatch results, FCXM produced the best results (83\% of the DSA detected). LXM outperformed the other tests in terms of the accuracy in separating class II DSA.
\end{abstract}

\section{Introduction}

The relevance of preformed human leukocyte antigen (HLA) antibodies in the context of transplantation was discovered in the 1960s [1]. Antibodies detected by Complement-dependent cytotoxicity (CDC) have the capacity to destroy donor cells via complement-mediated cytotoxicity. The fact that these antibodies have a clear effect on the cells attests to the clinical relevance of this assay for both screening and crossmatching [2]. However, accurate identification with CDC is problematic and the method is also very laborious. Innovations in commercial HLA antibody screening kits utilizing bead array technology have enabled the determination of antibody specificities with high accuracy and sensitivity $[3,4]$. This technology is now widely used and has replaced HLA antibody screening with the CDC method in many laboratories. The development of crossmatch techniques, however, has not made similar progress and consequently there are still many different crossmatch techniques in use today. This has led to a situation where crossmatching and screening are based on completely different technologies, quite often not even measuring the same variable.

As the main idea of a screening program is to predict crossmatches that would be negative, a screening method with differing sensitivity and specificity is far from ideal.
Predetermined antibody specificities allow virtual crossmatching to be performed as soon as the donor candidate has been typed for HLA. A virtual crossmatch performed with single antigen beads correlates with the graft outcome, but the correlation with a physical crossmatch is often not optimal due to differences in assays [5-7].

The predictive value of HLA antibodies identified with single antigen beads differs for each crossmatch method. Since FCXM, similarly to the routine bead array technique, identifies also non-complementbinding antibodies, it could be expected to produce more concordant results than Complement-dependent cytotoxicity crossmatch (CDCXM). However, false positive FCXM results are regularly seen and the crossmatch is not HLA-specific either [6]. One of the most recent crossmatch methods is a solid phase method where donor HLA molecules are captured on beads and analysed with a bead array. This method, LXM, is one of two HLA-specific crossmatch methods available, and it should correlate well with a virtual crossmatch [8]. The other available donor specific crossmatch, which is not evaluated in this study, is based on enzyme-linked immunosorbent assay [9].

\section{Objective}

Clinical laboratories working with organ transplantation struggle

Abbreviations: AUC, area under curve; CDC, Complement-dependent cytotoxicity; CDCXM, Complement-dependent cytotoxicity crossmatch; DSA, donor specific antibodies; DTT, dithiothreitol; FCXM, Flow cytometric crossmatch; LXM, Luminex crossmatch; MFI, Mean fluorescence intensity; ROC, receiver operating characteristic

* Corresponding author at: Histocompatibility Testing Laboratory, Finnish Red Cross Blood Service, Kivihaantie 7, FI-00310 Helsinki, Finland.

E-mail address: juha.perasaari@bloodservice.fi (J.P. Peräsaari). 
with the fact that antibody screening and the various crossmatching techniques differ greatly in terms of their sensitivity. The aim of this study was to compare the predictive value of the virtual crossmatch with various crossmatch techniques used in clinical laboratory settings and to identify the cut-off values with the best predictability.

\section{Materials and methods}

\subsection{Sample collection}

A total of 288 serum samples from 235 patients were used in crossmatching (the latest serum of each patient was used). Samples were stored frozen at $-20^{\circ} \mathrm{C}$. Crossmatching with different techniques was performed against splenocytes of 40 deceased donor candidates. For CDCXM, freshly separated cells were used. For the other crossmatching assays, splenocytes stored in liquid nitrogen were used.

\subsection{Donor HLA typing}

Donors were HLA typed with the complement-mediated lymphocytotoxicity test (Biotest Rockaway, $\mathrm{NJ}$ ) and a low-resolution polymerase chain reaction with sequence-specific primers (One Lambda Inc., Canoga Park, CA). Supplementary typing for HLA-A, B, C, DRB1-5, DQA1, DQB1, DPA1 and DPB1 was performed with a polymerase chain reaction with sequence-specific oligonucleotide probes (One Lambda) when the result was needed to confirm the donor-specificity of an antibody.

\subsection{HLA antibody screening}

Serum samples stored for routine crossmatching were used. Antibodies were screened and identified with Luminex-based commercial kits (LABScreen ${ }^{\circledR}$, One Lambda) from untreated serum samples. All specificities were identified with single antigen beads with a cut-off value of the baseline normalized value $1000 \mathrm{MFI}$ for an individual bead representing a single DSA. All sera were tested for HLA class I (HLA-A, $-\mathrm{B}$ and $-\mathrm{CW}$ ) and class II (HLA-DR, -DQ and -DP) antibodies. Antibodies were assigned with the HLA Fusion ${ }^{\mathrm{TM}}$ software v.3.2. DSA was determined by comparing assigned antibodies to the serological equivalent of the donor's HLA type. For anti-DQ and -DP antibodies, $\alpha$ and $\beta$ chain combinations were used in the analysis. Known Finnish haplotypes were utilised when a bead was selected to represent the antigen of the donor [10]. MFI values of the donor-specific antibodies were recorded. The sum of all individual DSAs above 1000 MFI was reported as the cumulative DSA [11,12].

\subsection{Complement-dependent cytotoxicity crossmatch}

CDCXM was performed during on-call with fresh density gradientpurified donor spleen cells using the technique by Amos [13]. Crossmatching was performed in parallel with various volumes of serum $(1 \mu \mathrm{l}, 5 \mu \mathrm{l}, 0.1 \mu \mathrm{l})$ as well as with an excess of complement (double amount). Serum and cells were incubated $20 \mathrm{~min}$ at room temperature and after complement addition $60 \mathrm{~min}$ at room temperature. Reactivity was scored according to the percentage of dead cells with the international workshop scoring: score 1: $<1 \%$; 2 : $1-20 \%$; $4: 21-50 \%$; 6 : 51-80\%; 8: $81-100 \%$. As a local modification, any cell death above background was considered positive (score 2). The highest reactivity of parallel testing was used as the strength of an individual crossmatch in further analysis. All crossmatches were performed without dithiothreitol (DTT).

\subsection{Flow cytometric crossmatch}

FCXM was performed retrospectively with frozen donor splenocytes [14]. T cell IgG and IgM and B cell IgG crossmatches were performed. In each test, 500,000 splenocytes were incubated with $50 \mu \mathrm{l}$ of serum for 30 min. After washing, T and B cells were identified with PE-anti-CD3 and PE-anti-CD19 (Cat no. 345765, 345777; BD Biosciences, San Jose, $\mathrm{CA})$. The secondary antibody was a FITC-conjugated $\mathrm{F}(\mathrm{ab})_{2}$ anti-human IgG (Cat no. 109-096-098 Jackson ImmunoResearch, West Grove, PA). After addition of fluorescence-labelled antibodies, cells were incubated for $15 \mathrm{~min}$ in the dark. Cells were analysed using FACScan instrument (BD Biosciences). A linear channel shift of at least 40 channels for $\mathrm{T}$ cells and 60 channels for B cells was considered positive.

\subsection{Luminex crossmatch}

LXM with Tepnel Donor specific antibody kit (currently Immucor Lifecodes) was performed retrospectively according to the manufacturer's protocol except for frozen splenocytes. Cells were thawed and washed to remove dimethyl sulfoxide and then lysed. For each test, a lysate containing $2.2 \times 10^{6}$ splenocytes was prepared. The lysate $(8 \mu \mathrm{l})$ was then incubated with $5 \mu \mathrm{l}$ of capture beads coated with either antiHLA class I or class II for $30 \mathrm{~min}$ in the dark at room temperature. These beads with captured donor HLA molecules where then incubated with $12 \mu \mathrm{l}$ of the patient serum for $30 \mathrm{~min}$ at room temperature in the dark. Binding of anti-HLA antibodies was detected with anti-human IgG conjugated with PE (R-phycoerythrin). Samples were run on LabScan 200 and analysed with the LifeMatch software (Tepnel, Lifecodes). A positive control bead (IgG) was used to verify the binding of anti-IgGPE. Three negative control beads were included and MFI values above 1000 against all three negative control values were considered positive.

\subsection{Statistical analysis}

Categorical variables were analysed with the Fisher's exact test. In the ROC analysis, also DSA values below $1000 \mathrm{MFI}$ were included as the analysis was used to determine the best cut-off value. The sensitivity and specificity of the various crossmatching methods were assessed with the ROC analysis. The accuracy of the test was classified as the AUC with 0.9-1: excellent; 0.8-0.9: good; 0.7-0.8: fair; 0.6-0.07: poor; 0.5-0.06: fail. Results with a $\mathrm{P}$ value of $\leq 0.05$ were considered statistically significant. Analyses were performed with the SPSS statistics version 21.0 software (IBM, Armonk, NY, USA).

\section{Results}

\subsection{HLA antibody profile}

Of the 364 crossmatches performed, the majority (68\% (246)) were performed with serum without HLA antibodies. Only $23 \%$ (83) of the crossmatches were performed against DSA. The mean cumulative DSA in DSA-positive crossmatches was 25,000 $\pm 23,000$ MFI, with the highest identified value being 90,000 MFI. Class I DSA was detected in $10 \%$ (37) and class II DSA in 5\% (17) of the crossmatches, while both class I and II DSA were detected in 8\% (29) of the crossmatches. Antibodies were most commonly directed against donor HLA-B antigens (13\% (48)) and least frequently against HLA-DRB3-5 (5\% (17)) and HLA-DP (5\% (17)) (Fig. 1).

\subsection{Complement-dependent cytotoxicity crossmatches}

Crossmatches were performed against donor splenocytes containing both T and B cells showing expression of Class I and Class II HLA antigens. A total of $30 \%(111 / 364)$ of the crossmatches were positive, with the highest scores being $2(26 \%), 4(35 \%), 6(17 \%)$ and $8(22 \%)$. DSA was present in only $50 \%(55 / 111)$ of the positive CDCXM (Table 1). A total of $11 \%(28 / 253)$ of the negative crossmatches were performed against DSA. The score for cell death in CDCXM correlated well with the degree of DSA positivity: for positive crossmatches with weak positivity (score 2) only $31 \%$ of the crossmatches were against 


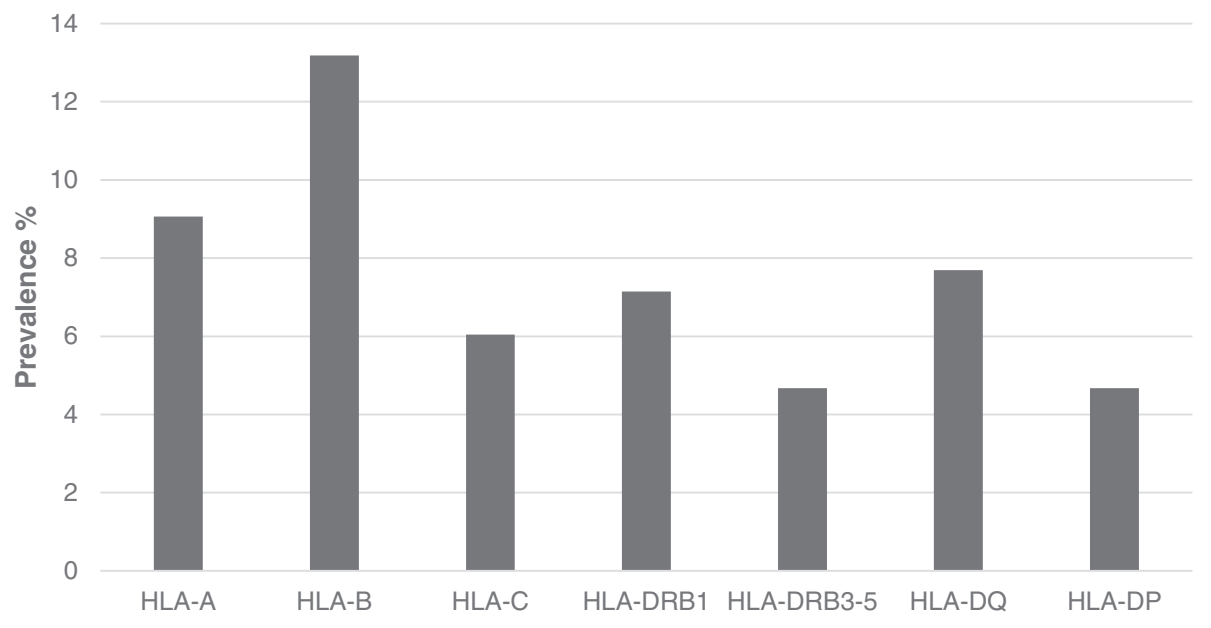

Fig. 1. Prevalence of DSAs directed against different HLA antigens in crossmatches. HLA, human leukocyte antigen; DSA, donor-specific antibody.
DSA, while for strong positives (score 8), 79\% of the crossmatches were against DSA. The total false positive and false negative rates for CDCXM with a routine cut-off (score 2$)$ were $20 \%(56 / 281)$ and $34 \%(28 / 83)$, respectively. When the cut-off was elevated to score 4 , the total false positive and false negative rates were $13 \%(36 / 281)$ and $45 \%(37 / 83)$, respectively.

\subsection{Flow cytometric crossmatch performance}

Separate crossmatches were performed on T and B cells in FCXM. Positivity was seen in $27 \%$ (97/364) of the crossmatches (Table 1$)$. The total true positive rate for FCXM was 83\% (69/83). In contrast, only 7\% $(19 / 275)$ of the negative crossmatches were performed against DSA. The total false positive and false negative rates were $10 \%(28 / 281)$ and $17 \%$ (14/83), respectively. T cells express only class I antigens, thus the $\mathrm{T}$ cell crossmatch was compared to the class I DSA status. With T cells, $78 \%(29 / 37)$ of the crossmatches performed only against class I DSA were positive. For Class II, the B cell crossmatch was comparable, with $76 \%(13 / 17)$ of the samples with only Class II DSA being positive. Interestingly, even with the higher class I expression in the splenic B cells, the B cell crossmatch did not perform better in Class I DSA detection than the $\mathrm{T}$ cell crossmatch $(68 \%$ vs $78 \%(\mathrm{p}=0.4328)$ Table 1$)$.

\subsection{Luminex crossmatch performance}

With LXM, it was possible to make separate crossmatches against HLA class I and class II antigens. In total, 71\% (59/83) of the crossmatches performed against DSA were positive (Table 1). The total false positive rate for LXM was $11 \%(31 / 281)$, while the false negative rate was $29 \%$ (24/83). The LXM test was able to distinguish between positive crossmatches due to class I and class II. For crossmatches with either class I or class II DSA only, the detection rate was $62 \%(23 / 37)$ and
$65 \%(11 / 17)$ respectively, while false positive rate was only seen in $5 \%$ $(15 / 298)$ of the class I and 7\% (22/318) of the class II crossmatches.

\subsection{Accuracy of different crossmatch techniques}

The accuracy of CDCXM in the identification of DSA at different cumulative MFI values was evaluated with ROC analysis. The sensitivity and specificity of different thresholds for CDCXM positivity are shown in Fig. 2A. The predictive accuracy of the test was fair (AUC: 0.724) when the routine threshold for positivity (score 2) was used. However, increasing the threshold of CDCXM to 4, 6 or 8 significantly improved the predictive accuracy from fair to good, with AUC being $0.748,0.802$ and 0.855 , respectively.

The performance of FCXM in the identification of any DSA (class I and/or class II) was good (AUC: 0.861) (Fig. 2B). Nearly equal performance was achieved with LXM (AUC: 0.805) (Fig. 2C).

\subsection{Crossmatching and separation of class I and II DSA}

A comparison of ROC analysis results for FCXM and LXM was performed as these are the only methods with the capacity to distinguish between crossmatches due to class I and class II antibodies. The limitation with CDCXM is that it is performed with splenocytes containing a mix of T and B cells. T cell FCXM and LXM showed similar diagnostic performance for class I DSA, with AUCs of 0.853 and 0.837, respectively (Fig. 2D). For class II DSA, both B cell FCXM and LXM reached a fair performance result as their AUC values were 0.740 and 0.775 , respectively (Fig. 2E).

\subsection{Sensitivity and specificity according to DSA level}

The effect of the cumulative DSA MFI value for each crossmatch

Table 1

Positive crossmatch results with different techniques and correlation with DSA

\begin{tabular}{|c|c|c|c|c|c|c|c|c|c|c|}
\hline & \multicolumn{2}{|c|}{$\mathrm{DSA}-(\mathrm{n}=281)$} & \multicolumn{2}{|c|}{$\mathrm{DSA}+(\mathrm{n}=83)$} & \multicolumn{2}{|c|}{ Only Class I DSA $(\mathrm{n}=37)$} & \multicolumn{2}{|c|}{ Only Class II DSA ( $\mathrm{n}=17$ ) } & \multicolumn{2}{|c|}{ Class I and II DSA $(\mathrm{n}=29)$} \\
\hline & $\mathrm{n}$ & $\%$ & $\mathrm{n}$ & $\%$ & $\mathrm{n}$ & $\%$ & $\mathrm{n}$ & $\%$ & $\mathrm{n}$ & $\%$ \\
\hline $\mathrm{CDCXM}^{*}$ & 56 & 20 & 55 & 66 & 21 & 57 & 10 & 59 & 24 & 83 \\
\hline T-cell FCXM & 20 & 7 & 61 & 73 & 29 & 78 & 7 & 41 & 25 & 86 \\
\hline B-cell FCXM & 25 & 9 & 64 & 77 & 25 & 68 & 13 & 76 & 26 & 90 \\
\hline Total FCXM & 28 & 10 & 69 & 83 & 29 & 78 & 13 & 76 & 27 & 93 \\
\hline Class I LXM & 13 & 5 & 41 & 49 & 23 & 62 & 2 & 12 & 16 & 55 \\
\hline Class II LXM & 20 & 7 & 30 & 36 & 2 & 5 & 11 & 65 & 17 & 59 \\
\hline Total LXM & 31 & 11 & 59 & 71 & 23 & 62 & 11 & 65 & 25 & 86 \\
\hline
\end{tabular}

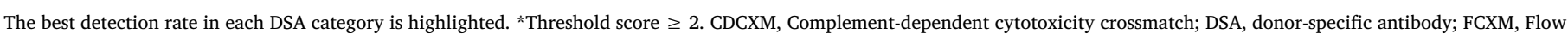
cytometric crossmatch; LXM, Luminex crossmatch. 

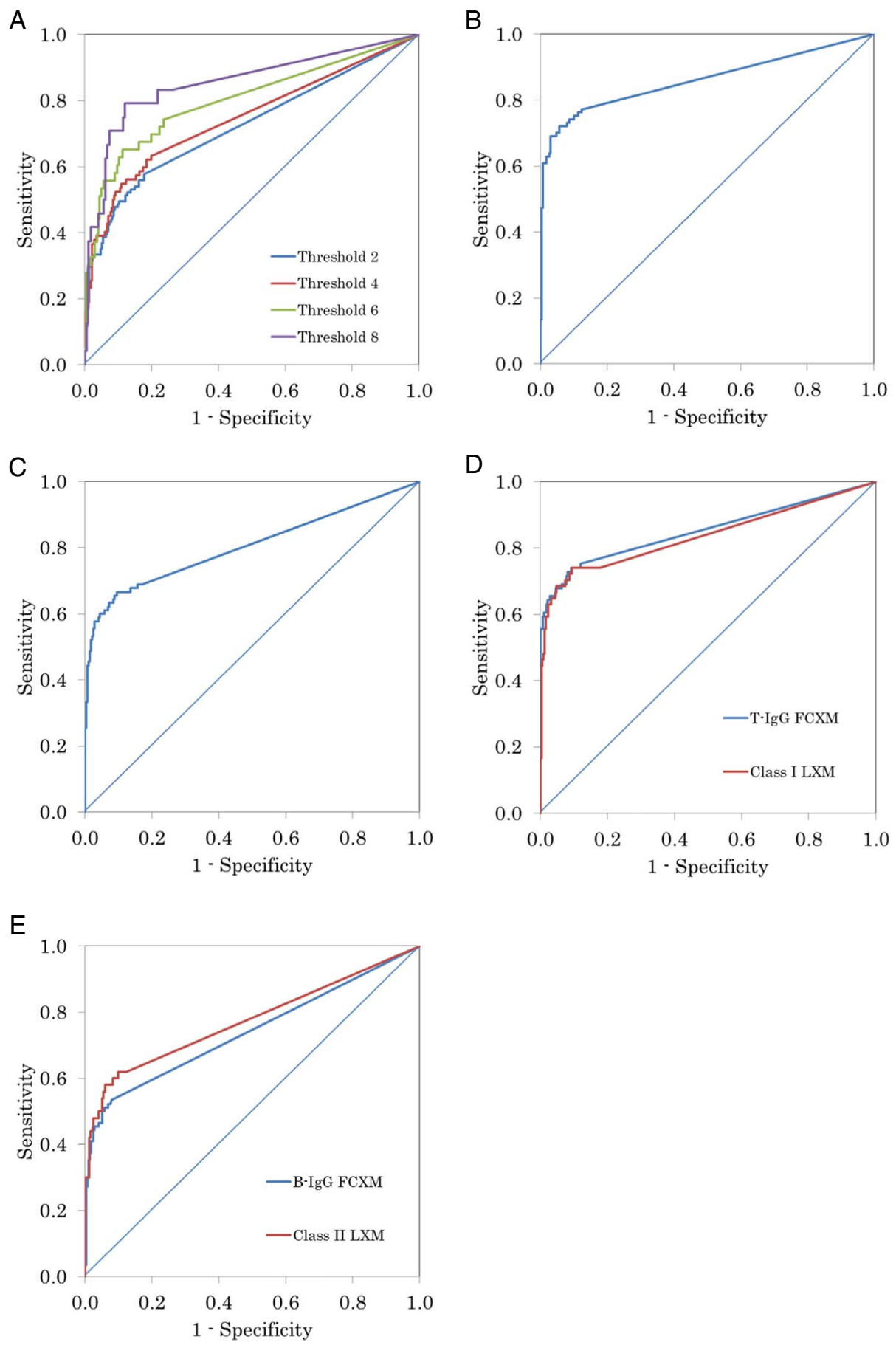

Fig. 2. ROC curve analysis of DSA in comparison with different crossmatch methods (A) DSA in comparison with different thresholds for CDCXM. Threshold 2: AUC 0.724; 4: AUC 0.748; 6: AUC 0.802; 8: AUC 0.855. (B) DSA in comparison with FCXM. AUC 0.861. (C) DSA in comparison with LXM. AUC 0.805. (D) HLA Class I DSA in comparison with FCXM and LXM AUC 0.853 and AUC 0.837, respectively. (E) HLA Class II DSA in comparison with FCXM and LXM AUC 0.740 and 0.775 , respectively. AUC $\geq 0.8$ was considered to constitute good accuracy for the test. The linear part represents repeated identical MFI values (no DSA identified). AUC, area under curve; CDCXM, Complement-dependent cytotoxicity crossmatch; DSA, donor-specific antibody; FCXM, Flow cytometric crossmatch; LXM, Luminex crossmatch; MFI, mean fluorescence intensity; ROC, receiver operating characteristic. 
A

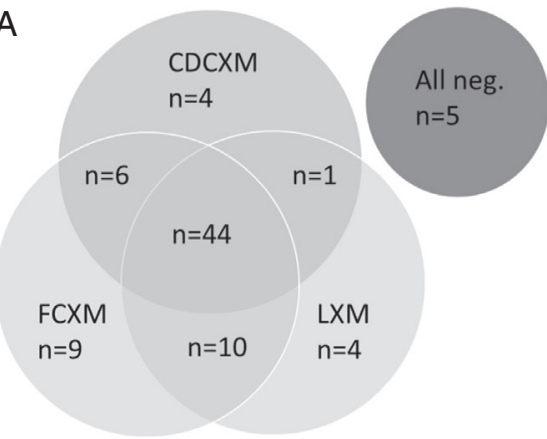

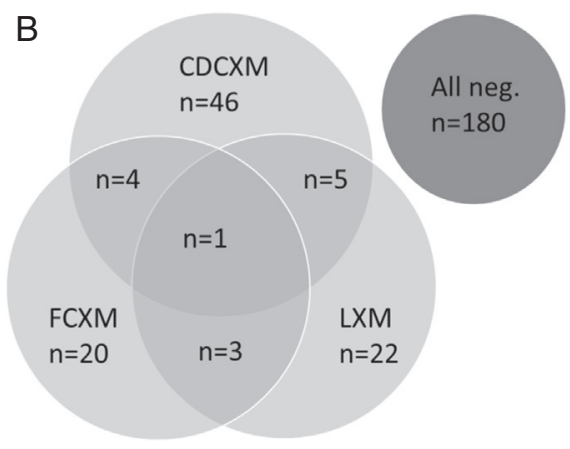

Fig. 3. Crossmatch-positivity of individual samples with each technique. (A) Crossmatches of DSA-positive samples. (B) Crossmatches of DSA-negative samples. method is shown in Table 2. The highest sensitivity in the identification of DSA can be reached with a cut-off of 1000 MFI with FCXM (0.722). Almost similar results can be obtained with LXM (0.667). The sensitivity of the assays rapidly decreases as the cut-off for the cumulative DSA increases. The specificity of the methods proved to be good. When the cumulative DSA MFI cut-off was adjusted, only a moderate effect on specificity was seen.

The cut-off resulting in the highest accuracy (the rate of true negative and true positive values) was different for each method. For FCXM, the best accuracy was achieved with both 1000 and 5000 MFI and for CDCXM with 5000 MFI, while LXM showed the highest accuracy with a cut-off of 10,000 MFI. The results of individual samples with each crossmatch method shows the concordance between the methods. From the crossmatches where DSA was present, only 6\% (5/ 83) were false negatives with all methods (Fig. 3A). However, most samples giving false positive results are different with each method (Fig. 3B).

\section{Discussion}

In this study, we compared a virtual crossmatch performed with single antigen beads to various crossmatch methods typically used in clinical laboratories serving organ transplant programs. The solid phase assay with single antigen beads has been proven to be sensitive and accurate in screening and identifying HLA antibodies. The drawback for the bead array is that it does not measure exactly the same variable as the crossmatch methods used routinely.

From the 83 crossmatches performed against DSA, the best performance was observed with FCXM (83\%). With LXM and CDCXM, the DSA detection rates were $71 \%$ and $66 \%$, respectively. The lower performance of CDCXM in detecting DSA is caused by lack of sensitivity [15]. This is partially due to the measurement of only antibodies capable of complement-dependent lymphocyte killing. In our study, the detection rate of $66 \%$ for DSA achieved with CDCXM is higher than reported in a recent study where the detection rate was $48 \%$ for $\mathrm{T}$ cells and $38 \%$ for B cells [16]. Also, the DSA detection rate of $83 \%$ with FCXM was higher than seen in previous studies in which the rates ranged from 52 to $65 \%[17,18]$. In our study, the better DSA detection rate could be explained by the fact that splenocytes were used as a source for donor cells. It is known that the density of HLA molecules especially in the B cell population from the spleen is higher than in blood-derived cells [19].

With the default cut-off of 1000 MFI for DSA, the highest false positive rate was seen for CDCXM (20\%). FCXM and LXM produced more accurate results ( $10 \%$ and $11 \%$, respectively). Based on our findings there is only a minor overlap on samples producing false negative and positive results between the methods. This indicates that the difference seen between the methods is based on the type of antibodies detected rather than the cut-off used in each technique. The high rate of false positives from CDCXM performed with a mixed $\mathrm{T}$ and $\mathrm{B}$ cell population is in line with a previous study where false positive rates of $18 \%$ and $23 \%$ were detected for $\mathrm{T}$ and $\mathrm{B}$ cells, respectively [16]. The false positive rate with the cytotoxic B cell crossmatch is known to be particularly high [20]. In our study, the higher number of false positives may be explained by IgM antibodies or autoantibodies as no DTT was used to neutralize these antibodies. In this study, 36\% (20/56) of the false positive CDCXMs were also positive for T cell IgM FCXM (data not shown). If this is taken into account, the rate for false positives would only be $13 \%(36 / 281)$ and the remainder of the false positives (7\% (20/ 281)) could be explained by IgM antibodies. Further, the prozone effect known to affect bead array technique may give false negative results as EDTA treatment was not used [21]. Therefore, some DSAs may have been missed. However, in our experience this phenomenon is rare in patients on transplant waiting list, yet it is seen more commonly in patients with immunopathological status.

The sensitivity and specificity of a crossmatch method depends largely on the cut-off value selected. It has been widely accepted that the sensitivity of FCXM is much better than that of CDCXM [22]. However, by adjusting the cut-off to avoid false positives, it is possible to decrease the sensitivity of a method even though the technique itself could perform better. In a clinical laboratory, the cut-off value needs to be adjusted to the consensus level defined by external proficiency testing. Further, the cut-off used must provide results with diagnostic value.

In organ transplantation, with a limited number of transplants, the tolerance for false negatives is low. Typically, laboratories aim at higher sensitivity at the expense of specificity in an effort to avoid hyperacute rejection and laborious treatment of antibody-mediated rejection. On the other hand, false positive crossmatches or crossmatches that are too sensitive prevent transplants with an acceptable outcome. We face this issue with the LXM technique as it is a new method and there is lack of experience in laboratory routine.

In our hands, the default cut-off for LXM was found to be too low because an unacceptably high number of false positives was detected. Thus, a laboratory-specific cut-off value was determined. However, this is based on a limited sample number and it may not be the most optimal. With the new cut-off, the method has the capability to separate crossmatch positivity due to class I and class II antibodies. Based on our results, it seems to be impossible to draw conclusions on class I or II positivity using $\mathrm{T}$ and $\mathrm{B}$ cell crossmatches only. Thus, $\mathrm{T}$ and $\mathrm{B}$ cell crossmatches are not equivalent for class I and II crossmatches. At 0.667 , the overall sensitivity of LXM was lower than reported earlier ( 0.96 for class I and 0.65 for class II) but the specificity of 0.905 was in line with that seen in previous studies ( 1.0 for class I and 0.97 for class II) [8]. Previous studies suggests that capture of DQ and DP molecules is impaired in the LXM method $[8,23]$. We were unable to confirm this finding as the number of samples with DSA against one loci only was very low as our samples were not selected to answer that question. Methods for detecting HLA class-specific antigens are scarce as most techniques detect any foreign antigens. However, LXM provides HLAspecificity as only donor HLA antigens are bound to the beads.

Generally, an AUC $\geq 0.8$ is considered to constitute a good test. If false negative results are considered detrimental, lower cut-off values producing fewer false negatives will not result in an AUC level required 
of a good test, as was evident with CDCXM. When the cut-off value was changed from score 2 to 8 , the accuracy of the test improved (AUC: 0.724-0.855). This result is in line with the findings of Wahrmann et al. [12] where similar results were obtained for class I and II separately.

The sensitivity of the crossmatch assays rapidly decreased when the cut-off for virtual crossmatch positivity was increased (table 2). However, the process is always a trade-off between sensitivity and specificity. If we use accuracy (rate of true positives and true negatives) as a standard for setting the cut-off value for the crossmatch techniques, a different cut-off should be set for each technique. With FCXM, the best accuracy was reached with cut-offs 1000 MFI and 5000 MFI while for CDCXM, the best accuracy was reached with the cut-off of 5000 MFI. For LXM, the cut-off value of 10,000 MFI was found to be the most optimal. However, the impact of the cut-off on accuracy was marginal, with a clear difference only seen for FCXM.

As long as routine screening methods identify antibodies based on their capacity to bind known targets it is practically impossible to detect the complete antibody repertoire of an individual serum. HLA class II antibodies present the greatest challenges as their targets are formed by alfa and beta chains produced by different genes. We have very little knowledge of the types of class II hybrid molecules that exist (e.g. DQA1 + DPB1) and of their relative proportions on donor cells. The target cells in CDCXM express also non-HLA antigens interfering with the test. Consequently, the virtual crossmatch is not equal to an actual crossmatch in performance.

Yet another limitation of the virtual crossmatch is the biological nature of the crossmatch and the unpredictability of the donor material. In a recent study by Badders et al. [19], it was shown that there is a broad variation in HLA expression between donors (for $\mathrm{T}$ cells from PBLs $455 \pm 160$ molecules of equivalent soluble fluorochromes). Also, extensive variation was seen between different cell sources (blood, spleen, node). Variation in HLA expression levels had a direct impact on the FCXM reactivity [19]. An antibody screening technique can be standardized but the density of HLA on the target cells cannot. Therefore, there will always be uncertainty unless HLA expression levels are assessed at the time of the crossmatch. The only crossmatch methods that permit the standardization of the amount of donor HLA are the methods in which donor antigens are captured onto a solid phase $[8,9]$.

\subsection{Limitations}

Some limitations apply to this study. The work could have been strengthened by correlation to clinical data. However, clinical data was not available for this study. In addition, only a small portion of the crossmatches resulted in transplantation, therefore a methodological approach was chosen. In CDCXM, only total lymphocytes were used in accordance with the national transplant protocol, thus no separate result for T and B cells were available. Also, the lack of DTT treatment results raises the false positive rate in CDCXM and hampers the comparability to results obtained by other laboratories.

\section{Conclusions}

To conclude, the best correlation was observed between the virtual crossmatch and FCXM, with LXM performing almost as well. This method seems particularly promising as it permits the standardization of the donor material, thereby producing even better correlation with the virtual crossmatch.

\section{Acknowledgements}

The authors thank the staff of the Histocompatibility Laboratory of the Finnish Red Cross Blood Service for their technical assistance. This work was supported by a grant from the Medical Research Fund of Finnish Red Cross Blood Service (EVO).

\section{References}

[1] R. Patel, P.I. Terasaki, Significance of the positive crossmatch test in kidney transplantation, N. Engl. J. Med. 280 (1969) 735-739.

[2] M.H. Christiaans, R. Overhof, A. ten Haaft, F. Nieman, J.P. van Hooff, E.M. van den Berg-Loonen, No advantage of flow cytometry crossmatch over complement-dependent cytotoxicity in immunologically well-documented renal allograft recipients, Transplantation 62 (1996) 1341-1347.

[3] A. Zeevi, A. Girnita, R. Duquesnoy, HLA antibody analysis: sensitivity, specificity, and clinical significance in solid organ transplantation, Immunol. Res. 36 (2006) 255-264.

[4] B.D. Tait, F. Hudson, L. Cantwell, et al., Review article: luminex technology for HLA antibody detection in organ transplantation, Nephrology (Carlton) 14 (2009) $247-254$.

[5] T.M. Ellis, J.J. Schiller, A.M. Roza, D.C. Cronin, B.D. Shames, C.P. Johnson, Diagnostic accuracy of solid phase HLA antibody assays for prediction of crossmatch strength, Hum. Immunol. 73 (2012) 706-710.

[6] E.K. Ho, E.R. Vasilescu, A.I. Colovai, et al., Sensitivity, specificity and clinical relevance of different cross-matching assays in deceased-donor renal transplantation, Transpl. Immunol. 20 (2008) 61-67.

[7] S. Riethmuller, S. Ferrari-Lacraz, M.K. Muller, et al., Donor-specific antibody levels and three generations of crossmatches to predict antibody-mediated rejection in kidney transplantation, Transplantation 90 (2010) 160-167.

[8] Billen EV, Voorter CE, Christiaans MH, Van den berg-Loonen EM. Luminex donorspecific crossmatches. Tissue Antigens 71 (2008) 507-513.

[9] C.W. Yang, Oh. EJ, S.B. Lee, et al., Detection of donor-specific anti-HLA class I and II antibodies using antibody monitoring system, Transplant. Proc. 38 (2006) 2803-2806.

[10] K. Haimila, J. Peräsaari, T. Linjama, et al., HLA antigen, allele and haplotype frequencies and their use in virtual panel reactive antigen calculations in the finnish population, Tissue Antigens 81 (2013) 35-43.

[11] J.P. Peräsaari, L.E. Kyllonen, K.T. Salmela, J.M. Merenmies, Pre-transplant donorspecific anti-human leukocyte antigen antibodies are associated with high risk of delayed graft function after renal transplantation, Nephrol. Dial. Transplant. 31 (2016) 672-678.

[12] M. Wahrmann, G. Hlavin, G. Fischer, et al., Modified solid-phase alloantibody detection for improved crossmatch prediction, Hum. Immunol. 74 (2013) 32-40.

[13] D.B. Amos, H. Bashir, W. Boyle, M. MacQueen, A. Tiilikainen, A simple micro cytotoxicity test, Transplantation 7 (1969) 220-223.

[14] I.H. Matinlauri, L.E. Kyllonen, B.H. Eklund, S.A. Koskimies, K.T. Salmela, Weak humoral posttransplant alloresponse after a well-HLA-matched cadaveric kidney transplantation, Transplantation 78 (2004) 198-204.

[15] H.M. Gebel, R.A. Bray, P. Nickerson, Pre-transplant assessment of donor-reactive, HLA-specific antibodies in renal transplantation: contraindication vs. risk, Am. J. Transplant. 3 (2003) 1488-1500.

[16] M. Alheim, P.K. Paul, D.M. Hauzenberger, A.C. Wikstrom, Improved flow cytometry based cytotoxicity and binding assay for clinical antibody HLA crossmatching, Hum. Immunol. 76 (2015) 849-857.

[17] N.L. Reinsmoen, J. Patel, J. Mirocha, et al., Optimizing transplantation of sensitized heart candidates using 4 antibody detection assays to prioritize the assignment of unacceptable antigens, J Heart Lung Transplant 35 (2016) 165-172.

[18] A.A. Zachary, J.T. Sholander, J.A. Houp, M.S. Leffell, Using real data for a virtual crossmatch, Hum. Immunol. 70 (2009) 574-579.

[19] J.L. Badders, J.A. Jones, M.E. Jeresano, K.P. Schillinger, A.M. Jackson, Variable HLA expression on deceased donor lymphocytes: not all crossmatches are created equal, Hum. Immunol. 76 (2015) 795-800.

[20] S. Le Bas-Bernardet, M. Hourmant, N. Valentin, et al., Identification of the antibodies involved in B-cell crossmatch positivity in renal transplantation, Transplantation 75 (2003) 477-482.

[21] M. Schnaidt, C. Weinstock, M. Jurisic, B. Schmid-Horch, A. Ender, D. Wernet, HLA antibody specification using single-antigen beads-a technical solution for the prozone effect, Transplantation 92 (2011) 510-515.

[22] H.M. Gebel, R.A. Bray, Sensitization and sensitivity: defining the unsensitized patient, Transplantation 69 (2000) 1370-1374.

[23] J.L. Caro-Oleas, M.F. Gonzalez-Escribano, S. Toro-Llamas, et al., Donor-specific antibody detection: comparison of single antigen assay and luminex crossmatches, Tissue Antigens 76 (2010) 398-403.

\section{Conflict of interest}

